\title{
Observation of oscillations between degenerate bound-state configurations in rapidly autoionizing two-electron atoms
}

\author{
M. B. Campbell, T. J. Bensky, and R. R. Jones \\ Department of Physics, University of Virginia, Charlottesville, Virginia 22901
}

\begin{abstract}
The oscillation between bound-state configurations in a rapidly autoionizing three-body Coulomb system has been directly observed. Using a 500-fsec laser pulse, calcium atoms are excited to the pure $4 p_{3 / 2} 15 d$ twoelectron configuration at an energy greater than $3 \mathrm{eV}$ above the ionization limit. As a result of configuration interaction, the electrons scatter coherently into multiple bound and continuum configurations. The oscillation between the degenerate $4 p_{1 / 2} n^{\prime} d$ and $4 p_{3 / 2} n d$ modes as well as autoionization into $4 s_{1 / 2} \epsilon \ell, 3 d_{3 / 2} \epsilon \ell$, and $3 d_{5 / 2} \epsilon \ell$ continua are observed explicitly using bound-state interferometry. The measured time dependence of the $4 p_{3 / 2} 15 d$ character is in excellent agreement with the Fourier transform of the frequency domain excitation cross section. To our knowledge, this is the first experimental demonstration of the equivalence of time and frequency domain spectra in a multiconfigurational system involving bound and continuum channels.
\end{abstract}

\section{INTRODUCTION}

Because of its combined conceptual simplicity and inherent complexity, the three-body Coulomb system, two optically active electrons bound to a doubly charged optically inactive ion core, has held great interest for decades. Because of their mutual repulsion, each electron moves in a noncentral potential. Therefore, energy and angular momentum are conserved quantities for the entire system, but not for each electron individually. The eigenstates of the system, in both the discrete and continuous parts of the spectrum, are linear superpositions of products of different single-electron configurations. The eigenstates are said to exhibit configuration mixing due to the electron-electron Coulomb repulsion or "configuration interaction.', Historically, experimental and theoretical studies of these three-body Coulomb systems have concentrated on the recovery of time-independent parameters to describe the eigenstates of the atom as a function of energy and angular momentum [1]. The multichannel quantum defect theory (MQDT) formalism has proved to be invaluable in this regard [2].

In spite of the success of the frequency domain approach for describing nominally two-electron atoms, semiclassical scattering is often invoked to provide a more accessible physical picture of the electron dynamics [1]. In such a timedomain description, both classical electrons have a welldefined energy and angular momentum at any given time. The electrons exchange energy and angular momentum during collisions. The relative orientation and proximity of the electrons to each other and the nucleus during a collision determines their new energies and momenta. The amplitude of the various dielectronic configurations in the frequencydomain eigenstates is directly related to the amount of time that a classical electron pair spends in each accessible configuration.

Using coherent short-pulse excitation, it is now possible to localize a two-electron atom in a single configuration that is not an eigenstate of the system [3]. The electron configu- ration in the atom is then a time-dependent quantity that can be monitored as the system evolves. The transfer of electron population between different configurations is made possible through the Coulomb interaction during collisions. Therefore, using time-domain methods, one can study intra-atomic electron scattering directly, taking full advantage of semiclassical insight. Furthermore, these time-domain methods make it possible to localize quantum probability in one of many energetically accessible configurations or modes at specific times. This capability is crucial to the concept of quantum control [4].

This report describes experiments in which short-pulse laser excitation is used to produce excited-state atoms in a single configuration at an energy greater than $3 \mathrm{eV}$ above the first ionization limit in calcium. At this energy, the configuration interaction rapidly transfers population to many other bound and unbound dielectronic modes. Bound-state interferometry [5-7] is used to monitor the electron probability in the initial bound configuration as a function of time. We observe autoionization via scattering into unbound channels as well as coherent scattering between bound modes. Complete depletion and then revival of the initial-state configuration is observed during the autoionization lifetime. The time-domain observations are compared to a discrete Fourier transform of the frequency-domain excitation cross section of the initial nonstationary state. Quantitative agreement between the two methods for obtaining the temporal-state evolution is achieved. This is the first demonstration of the equivalence of the time- and frequency-domain approaches for describing time-dependent configuration interaction in a complicated system with multiple bound and continuum channels.

\section{EXPERIMENTAL PROCEDURE}

The experiment takes place in a vacuum system with a background pressure of $10^{-6}$ Torr. A fraction of the $4 s^{2}{ }^{1} S_{0}$ ground-state calcium atoms in an effusive beam are excited through an intermediate $4 s 4 p{ }^{1} P_{1}$ level to the $4 s 15 d{ }^{1} D_{2}$ 


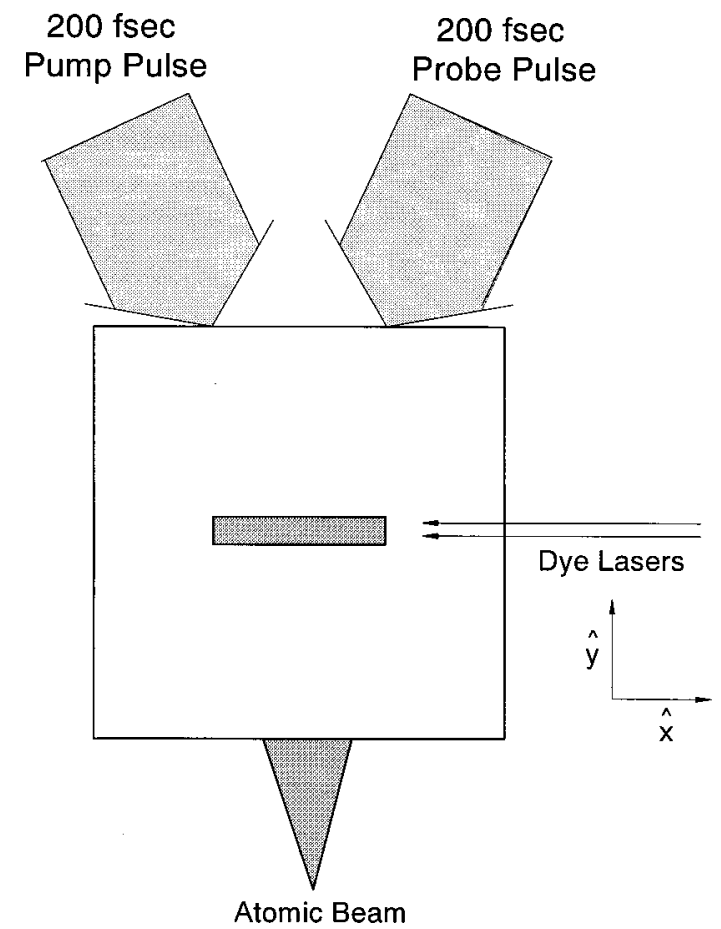

FIG. 1. Schematic diagram of the experimental apparatus showing the atomic, dye laser, and short-pulse laser beams. The angle between the propagation directions of the two short pulses is greatly exaggerated.

Rydberg eigenstate using two nanosecond dye lasers that propagate at right angles to the atomic beam (see Figs. 1 and 2). Next, the atoms are exposed to a 200 -fsec, 393-nm laser pulse which drives an isolated core excitation (ICE) [8] of the $4 s$ electron to produce atoms in a nearly pure $4 p_{3 / 2} 15 d$ autoionizing state. This level lies $24866 \mathrm{~cm}^{-1} \approx 3.1 \mathrm{eV}$ above the first ionization limit of $\mathrm{Ca}$.

In the time domain, the brief duration of the laser pulse

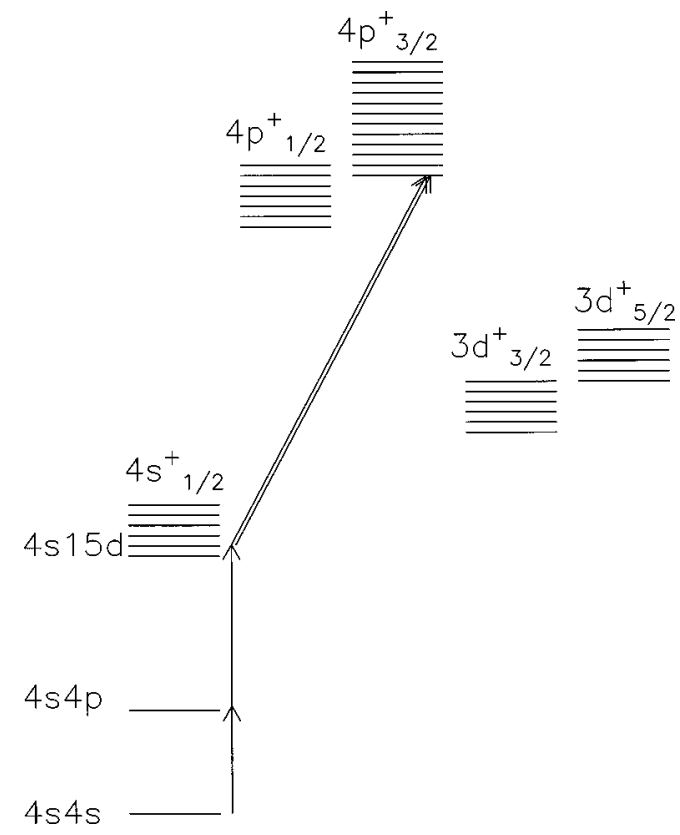

FIG. 2. Calcium energy-level diagram showing the neutral and ionic levels of particular interest to this experiment. ensures that negligible configuration mixing occurs during the pulse, so that the excited amplitude is in a pure $4 p_{3 / 2} 15 d$ mode. In the frequency domain, the $\approx 75-\mathrm{cm}^{-1}$ coherent bandwidth of the laser excites the $4 p_{3 / 2} 15 d$ character over a range of energies, producing a nonstationary two-electron wave packet. After the pulse, the wave packet scatters into many degenerate bound and continuum dielectronic configurations. These modes can be identified as singly and doubly excited channels whose Rydberg series converge to at least five different ionic core levels including $\mathrm{Ca}^{+} 4 s_{1 / 2}, 3 d_{3 / 2}$, $3 d_{5 / 2}, 4 p_{1 / 2}$, and $4 p_{3 / 2}$ (see Fig. 2).

Theoretically, one could learn everything about the electron dynamics by probing the time dependence of the population amplitude in each two-electron configuration. Unfortunately, this measurement is not experimentally possible with current technology. However, a wealth of dynamical information can be obtained by monitoring the population remaining in the initial $4 p_{3 / 2} 15 d$ configuration as a function of time. It is straightforward to make this measurement using bound-state interferometry or the optical Ramsey method [5-7]. The method has been described in detail elsewhere [9], and so only a brief discussion is given here. After the initial ICE, a second identical 200-fsec laser pulse coherently excites another bundle of probability amplitude directly into the $4 p_{3 / 2} 15 d$ configuration. The excited-state wave packet produced by the second pulse interferes with that produced by the first pulse, causing a modulation in the total autoionization yield. The interference level depends on the relative delay $\tau$ between the two identical pulses and is a direct measure of the spatial overlap between the two nonstationary states during the second pulse. Since the second pulse excites probability amplitude into the $4 p_{3 / 2} 15 d$ configuration only, the interference level reflects the $4 p_{3 / 2} 15 d$ character of the first wave packet after a time delay $\tau$. Therefore, the evolution of the $4 p_{3 / 2} 15 d$ character can be monitored by measuring the total autoionization yield as a function of the relative delay $\tau$ between the 200 -fsec laser pulses.

Almost invariably, the time scale for motion of Rydberg wave packets is orders of magnitude longer than the period of the wave packet interference which occurs at the optical period of the light field in the short pulses. Consequently, an enormous amount of data is required if one wishes to record the changes in the ionization signal at both the optical and wave packet periods [7]. A variety of different experimental techniques have been used to reduce the amount of data required to implement the optical Ramsey method without significantly limiting its information content.

First, nonlinear root-mean-square (rms) averaging allows one to sample interferograms using delay steps that are large compared to the optical period, but small compared to the wave packet time scales of interest. In this method, the wave packet interference appears as signal noise [6]. It is very effective for picking out temporal features in the true interferogram, but it can produce relatively large uncertainties in the relative amplitudes of the various features. Since the interference signal is indistinguishable from other noise sources, time-dependent variations in the background noise level and beating between the sample and optical interference periods can produce artificial structure in the interferograms.

Second, the two optical pulses can be phased locked so 
that the interference signal is only sampled at a well-defined phase difference between the two optical pulses [5]. This method eliminates the dominant noise sources that occur in the rms technique while reducing the amount of data that must be collected. Unfortunately, the phase-locked technique requires an additional high-resolution spectrum analyzer or simultaneous measurement of the phase delay in a cw reference laser. Therefore, it is rarely used.

Third, a combination of micro- and macrosample steps can be used to monitor the interference as a function of delay without sampling the complete interferogram [10]. In this method, data are first sampled with very fine steps over a few interference oscillations. Next, the delay between the two pulses is increased with a macrostep that is large compared to the optical period, but small compared to the time scale for electron motion. The data are then sampled with small, high-resolution, time steps again. This method does not suffer from the limitations of the rms or phase-locked techniques, but it requires that more data be collected than either of these other options.

In spite of the fact that each of these techniques allows data to be acquired at an increased rate, the destructive nature of conventional pump-probe techniques limits the information obtained with each laser shot to the state of the atom at a single pump-probe delay. Therefore, the data collection process is relatively slow and requires negligible equipment drift during long delay runs. To circumvent this problem, we have developed a new detector that enables us to acquire pump-probe data over a range of delays with a single laser shot [11]. The principle of operation behind the single-shot detector is similar to that of optical cross-correlators that are commonly used to measure the temporal profile of ultrashort laser pulses. In the current experiment, the detector is used to collect delay data over several optical interference periods using a single laser shot. It is then possible to implement the micro- and macrostep technique [10] while only physically taking macrodelay steps.

A single $787-\mathrm{nm}, \approx 1$ - $\mathrm{mJ}$ pulse from an amplified, 150fsec Ti:sapphire laser is used to generate the two phaselocked 200-fsec, 393-nm laser pulses required for the interferometric measurement. A Michelson interferometer is used to split the 787-nm pulse into two identical pulses with a well-defined, but variable delay. The interferometer is slightly misaligned so that the two pulses leave the interferometer with a small angle $\theta$ between their directions of propagation. Both pulses are sent through a $1 \mathrm{~mm} \times 10 \mathrm{~mm} \times 10 \mathrm{~mm}$ KDP crystal producing two $393-\mathrm{nm}$, 200 -fsec laser pulses. The 393-nm pulses enter the vacuum chamber and propagate through the interaction region antiparallel to the atomic beam. The diameters of the atomic and laser beams are all approximately $1 \mathrm{~cm}$ in the interaction region. Because the 200-fsec pulses are not exactly collinear (shown greatly exaggerated in Fig. 1), the relative delay between the two pulses varies linearly along the $\hat{x}$ axis. The difference in the relative delay between the pump and probe pulses, as seen by two atoms separated by a distance $d$ along the $\hat{x}$ axis, is $\Delta \tau=2 d \sin (\theta / 2) / c$, where $c$ is the speed of light. Therefore, atoms which experience a continuous range of pump-probe delays are produced with each laser shot, and the number of ions produced as a function position along the $\hat{x}$ axis reflects the $4 p_{3 / 2} n d$ excitation probability as a func-

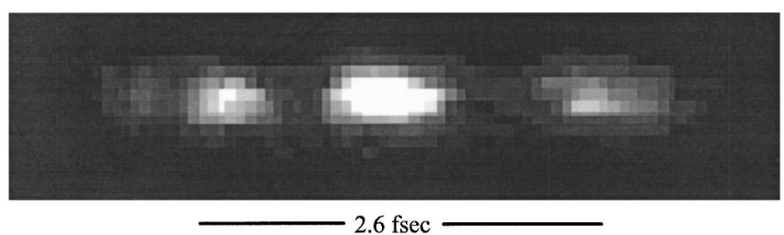

FIG. 3. Raw video image of autoionization signal versus position within the interaction region. The relative delay between the two 200 -fsec laser pulses changes by approximately 5 fsec across the full image.

tion of the relative delay between the two 393-nm pulses.

The laser and atomic beams overlap between two $7.5 \mathrm{~cm} \times 7.5 \mathrm{~cm}$ parallel capacitor plates that are separated by $1.5 \mathrm{~cm}$. The long dimension of a $0.25 \mathrm{~cm} \times 1.5 \mathrm{~cm}$ slit is oriented parallel to the $\hat{x}$ axis in the upper capacitor plate. Approximately $20 \mathrm{nsec}$ after the short pulses interact with the atoms, $a \approx 50-\mathrm{V}$ pulse applied to the lower field plate pushes any ions formed by autoionization through the slit toward a microchannel plate (MCP) detector. The ions strike the channel plates with their relative positions along the $\hat{x}$ axis preserved. The secondary electrons formed in the channel plates are accelerated onto a phosphor screen mounted on the top of the channel plate assembly. The spatial distribution of ions in the interaction region can be determined after each laser shot by monitoring the phosphor fluorescence level with a charged-coupled diode (CCD) camera. The spatial resolution of the combined phosphor and MCP is approximately $300 \mu \mathrm{m}$. The angle between the two 393-nm beams is chosen to give a maximum delay of approximately $5 \mathrm{fsec}$ across the atomic beam with better than $0.05 \mathrm{fsec}$ resolution. In this geometry, approximately three full Ramsey interference oscillations can be seen in the autoionization signal after a single shot [12]. Figure 3 shows the raw video image from a single 787-nm laser shot. Three maxima in the ionization signal can be clearly seen.

After each laser shot, a digital oscilloscope is used to capture a single line of the video signal corresponding to the center of the extraction slit. This line image is averaged over several laser shots to improve the signal-to-noise ratio. The optical path length in the Michelson interferometer is then increased by $10 \mu \mathrm{m}$ to produce a macrodelay step of $33 \mathrm{fsec}$. Since the electronic interference appears at the optical frequency, the interference period in the line image is a constant that depends only on the angle $\theta$ between the short-pulse propagation directions. Therefore, the strength of the sinusoidal interference pattern at each macrostep can be obtained using a discrete Fourier transform of the video line image. The amount of $4 p_{3 / 2} 15 d$ character in the wave packet at a given macrostep delay is proportional to the power within a narrow frequency window of the transformed image. In this way, the data are automatically noise filtered, enhancing the signal-to-noise ratio.

\section{DISCUSSION}

Figure 4 shows the Ramsey interference amplitude as a function of delay for an ICE of the $4 p_{3 / 2} 15 d$ level. The data are averaged over four different macrostep delay scans. If the $4 p_{3 / 2}$ nd configuration were the only bound configuration in 


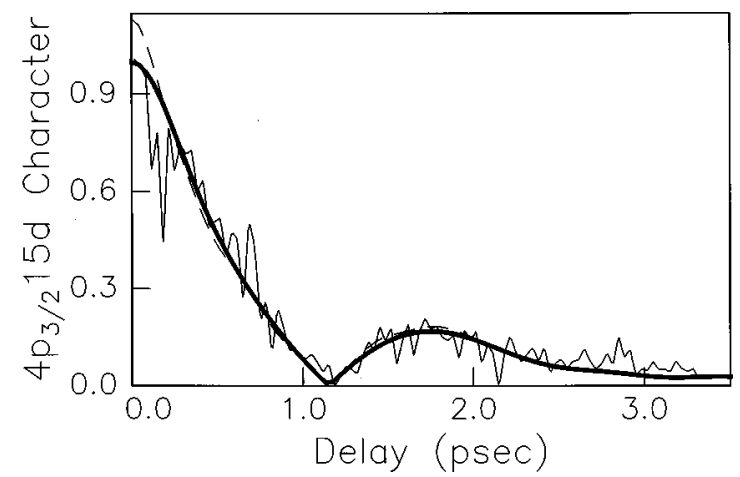

FIG. 4. Time dependence of the $4 p_{3 / 2} 15 d$ character in the autoionizing wave packet. The noisy light solid line is obtained from a measurement of the Ramsey interference level using bound-state interferometry. The relatively high noise level for delays less than $200 \mathrm{fsec}$ is due to amplitude modulation of the 787-nm pulse at the output of the Michelson interferometer. The dashed line is a discrete Fourier transform of the full $4 p_{3 / 2} 15 d$ ICE spectrum shown in Fig. 5. The bold solid curve is obtained by Fourier transforming the product of the ICE spectrum and the 200-fsec laser spectrum shown in Fig. 5. Note that the finite bandwidth of the 200 -fsec pulse only changes the interference amplitude near $t=0$.

the problem, an exponential decrease in the interference amplitude, corresponding to autoionization into the $4 s$ and $3 d_{j}$ continua, would be observed. The clear deviation from exponential decay is due to coherent coupling of the $4 p_{3 / 2}$ nd channel with the degenerate $4 p_{1 / 2} n^{\prime} d$ configuration. For the case $n=15$, the dominant interference comes from scattering into states with $n^{\prime}=19$ and 20 .

At $t=0$, the wave packet is in a pure $4 p_{3 / 2} 15 d$ state. However, scattering between the two electrons rapidly spreads the character among other bound and continuum channels. The coherent scattering is complete after only 1.2 psec when zero population remains in the initial configuration [13]. In a continuum configuration one electron rapidly leaves the vicinity of the atom, and so no rescattering into bound modes can occur. However, the bound $4 p_{1 / 2} n d$ population that survives the continued autoionization loss can be transferred back into the $4 p_{3 / 2} 15 d$ state. A $20 \%$ revival in the initial configuration is observed near 1.8 psec. Further bound-state oscillations are suppressed by autoionization, which is nearly complete after only 3.0 psec or so.

As discussed in the Introduction, both time-domain and frequency-domain descriptions of the atom should be correct. Figure 4 gives a clear, relatively simple view of the effects of the time-dependent configuration interaction during the wave packet's evolution. Can a simple picture be obtained in the frequency domain? Figure 5 shows the cross section for ICE of the $4 p_{3 / 2} 15 d$ level using a $393-\mathrm{nm}$ pulse with a duration of $5 \mathrm{nsec}$ and a bandwidth of $\approx 1 \mathrm{~cm}^{-1}$ [14]. The excitation line shape is not Lorentzian and indicates interference between multiple bound Rydberg channels converging to the $4 p_{1 / 2}^{+}$and $4 p_{3 / 2}^{+}$ionic levels. Although $a b$ initio $R$-matrix calculations could be used to reproduce this spectrum, neither the spectrum nor the $R$-matrix elements supply the same instantaneous information regarding the dynamic electron-electron scattering.

However, it has been shown experimentally and theoreti-

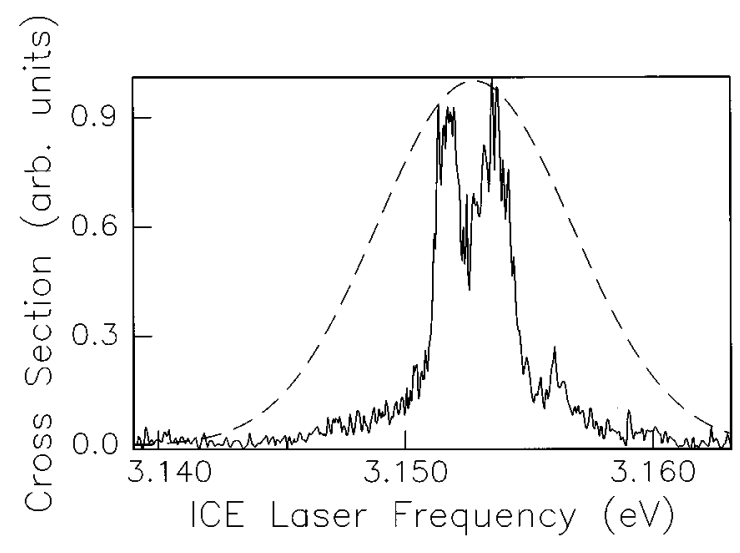

FIG. 5. Frequency dependence for ICE of the $4 p_{3 / 2} 15 d$ autoionizing state as a function of the frequency of a narrow-band 5-nsec laser pulse. The smooth dashed curve is a Gaussian that approximates the spectrum of the $200-$ fsec laser pulses used in the timedomain experiments.

cally that the Fourier transform of the frequency-domain excitation spectrum is equivalent to the time-domain boundstate interferogram [15]. The theoretical formulation of that equivalence does not restrict the excitation to pure bound states. Nevertheless, to the best of our knowledge, no experimental verification of this equivalence has been successfully demonstrated for multiconfigurational autoionizing states. Because of the relatively high signal to noise in the frequency-domain spectrum and time-domain interferogram presented here, we are in a unique position to quantitatively test that prediction.

The dashed curve in Fig. 4 is obtained by taking a discrete Fourier transform of the frequency domain spectrum from Fig. 5. Note that this line does not agree perfectly with the interferogram at short delays near $t=0$. This small discrepancy is due to a breakdown in the fundamental assumption of our time-domain study. Namely, we assumed that the interferogram gave the time-dependent $4 p_{3 / 2} 15 d$ character in the wave packet. However, this assumption is only perfectly correct if pulses with infinitely short duration are used. The finite, 200-fsec duration of our pulses means that the character of other channels can be found in the wave packet at $t=0$ due to configuration interaction during the pulse. The true time dependence of the $4 p_{3 / 2} 15 d$ character is given by the Fourier transform of the frequency-domain spectrum, which, for all practical purposes, has an infinite bandwidth. We can simulate the effect of finite pulse duration on the interferogram by multiplying the frequency-domain excitation spectrum by the spectrum of the $200-$ fsec laser pulses (see the dashed line in Fig. 5) and then Fourier transforming. The resulting dark curve shown in Fig. 4 is in excellent agreement with the time-domain measurement.

\section{SUMMARY}

We have used bound-state interferometry and a new single-shot detector to monitor the dielectronic configuration interaction in doubly excited states of calcium. The interferogram clearly shows scattering into continuum and bound channels. The coherent bound-state mixing produces a pro- 
nounced oscillation in the bound-state character. This is the first observation of beating between bound-state configurations due to electron-electron scattering in a system that is strongly damped by autoionization. The observation of complete population inversion from one mode to another in the presence of damping is an important step in proposed schemes for quantum control in multiconfigurational systems [4]. In addition, our time-domain data are compared to the Fourier transform of the frequency-domain excitation spectrum. The results of this experiment show quantitative agree-

[1] T. F. Gallagher, Rydberg Atoms, 1st ed. (Cambridge University Press, Cambridge, England, 1994).

[2] U. Fano, Phys. Rev. A 2, 353 (1970); M. J. Seaton, Rep. Prog. Phys. 46, 167 (1983); W. E. Cooke and C. L. Cromer, Phys. Rev. A 32, 2725 (1985).

[3] D. W. Schumacher, D. I. Duncan, R. R. Jones, and T. F. Gallagher, J. Phys. B 29, L1 (1996); D. W. Schumacher, B. J. Lyons, and T. F. Gallagher, Phys. Rev. Lett. 78, 4359 (1997); B. J. Lyons, D. W. Schumacher, D. I. Duncan, R. R. Jones, and T. F. Gallagher, Phys. Rev. A 57, 3712 (1998).

[4] D. J. Tannor and S. A. Rice, J. Chem. Phys. 83, 5013 (1985); W. S. Warren, H. Rabitz, and M. Dahleh, Science 259, 1581 (1993).

[5] N. F. Scherer et al., J. Chem. Phys. 93, 856 (1990); L. D. Noordam, D. I. Duncan, and T. F. Gallagher, Phys. Rev. A 45, 4734 (1992).

[6] B. Broers, J. F. Christian, J. H. Hoogenraad, W. J. van der Zande, H. B. van Linden van den Heuvell, and L. D. Noordam, Phys. Rev. Lett. 71, 344 (1993).

[7] R. R. Jones, C. S. Raman, D. W. Schumacher, and P. H. Bucksbaum, Phys. Rev. Lett. 71, 2575 (1993).

[8] W. E. Cooke, T. F. Gallagher, S. A. Edelstein, and R. M. Hill, ment between these two approaches when continuum states are involved.

\section{ACKNOWLEDGMENTS}

We gratefully acknowledge stimulating discussions with D. W. Schumacher and T. F. Gallagher and the financial support of the Office of Naval Research, the National Science Foundation, the Packard Foundation, and the University of Virginia Academic Enhancement Program.

Phys. Rev. Lett. 40, 178 (1978)

[9] L. D. Noordam and R. R. Jones, J. Mod. Opt. 44, 2515 (1997), and references therein.

[10] M. W. Noel and C. R. Stroud, Jr., Phys. Rev. Lett. 77, 1913 (1996).

[11] M. B. Campbell, T. J. Bensky, and R. R. Jones, Optics Express 1, 197 (1997).

[12] The single-shot detector can be used in virtually any type of pump-probe experiment. By increasing the angle between the direction of propagation of the pump and probe beams from near 0 to $\pi$, the temporal resolution of the detector can be adjusted from subfemtosecond to picosecond as required for a particular experiment.

[13] A constant background is subtracted from the data shown in Fig. 4 to compensate for the small amount of residual noise that is present at the Ramsey interference frequency even in the absence of any real interference signal. Since autoionization ensures that the bound-state character is negligible at long times, the constant background is chosen accordingly.

[14] R. R. Jones, Phys. Rev. A (to be published).

[15] R. R. Jones, D. W. Schumacher, T. F. Gallagher, and P. H. Bucksbaum, J. Phys. B 28, 405 (1995). 